\title{
Fear of cancer recurrence following allogeneic haematopoietic stem cell transplantation (HSCT) for haematological malignancy: A cross-sectional study
}

\author{
Lisa Brice $^{\text {a }}$, Gemma McErlean ${ }^{\text {b,c,d, }}$, Caroline Donovan ${ }^{\mathrm{e}}$, Caley Tapp ${ }^{\mathrm{e}}$, Nicole Gilroy ${ }^{\mathrm{b}}$, \\ Masura Kabir ${ }^{\mathrm{f}}$, Matt Greenwood ${ }^{\mathrm{a}, \mathrm{g}}$, Stephen R. Larsen ${ }^{\mathrm{h}}$, John Moore ${ }^{\mathrm{i}}$, David Gottlieb ${ }^{\mathrm{j}}$, \\ Mark Hertzberg ${ }^{\mathrm{k}}$, Louisa Brown ${ }^{1}$, Megan Hogg ${ }^{j}$, Gillian Huang ${ }^{j}$, Jeff Tan ${ }^{\mathrm{i}}$, \\ Christopher Ward ${ }^{\text {a,c,f }}$, Ian Kerridge ${ }^{a, c, f}$
}

${ }^{a}$ Department of Haematology, Royal North Shore Hospital, Sydney, NSW, Australia

${ }^{\mathrm{b}}$ Blood and Marrow Transplant Network, New South Wales Agency for Clinical Innovation, Sydney, NSW, Australia

${ }^{\mathrm{c}}$ Northern Clinical School, Faculty of Medicine, University of Sydney, Sydney, NSW, Australia

d Graduate School of Health, University of Technology, Sydney, NSW, Australia

e School of Applied Psychology, Griffith University, QLD, Australia

${ }^{\mathrm{f}}$ Westmead Breast Cancer Institute, Sydney, NSW, Australia

${ }^{g}$ Northern Blood Research Centre, Kolling Institute, University of Sydney, NSW, Australia

${ }^{\mathrm{h}}$ Institute of Haematology, Royal Prince Alfred Hospital, Sydney, NSW, Australia

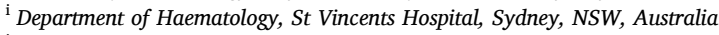

${ }^{\mathrm{j}}$ Department of Haematology, Westmead Hospital, Sydney, NSW, Australia

${ }^{\mathrm{k}}$ Department of Haematology, Prince of Wales Hospital, Sydney, NSW, Australia

${ }^{1}$ Calvary Mater Hospital, Newcastle, NSW, Australia

\section{A R T I C L E I N F O}

\section{Keywords:}

Blood and marrow transplant

Haematopoietic stem cell transplantation

Cancer survivors

Fear of cancer recurrence

Haematological malignancy

Psychological functioning

Quality of life

\begin{abstract}
A B S T R A C T
Purpose: The aim of this study was to quantify the prevalence of Fear of Cancer Recurrence (FCR) in patients with a prior haematology malignancy surviving more than one year post allogeneic haematopoietic stem cell transplantation (HSCT), and to identify the demographic, medical and psychological factors associated with FCR occurrence.

Method: Participants were adult allogeneic HSCT recipients who had undergone the procedure for acute leukaemia or other haematological malignancy between the years 2000-2012 in Sydney, Australia. They completed a purpose designed survey and six other validated instruments which assessed FCR, psychological functioning, quality of life, demographic, social and clinical variables.

Results: Of the 364 respondents, approximately 11\% of the sample lived with severe FCR while only 5\% of subjects reported having no FCR. Variables significantly associated with higher FCR included unemployment, a shorter time (years) post-transplant, not attending to health screening (PAP smear), a secondary diagnosis of skin cancer, younger age, referral to a psychiatrist and taking psychotropic medication. Higher psychological distress (depression, anxiety, stress) and lower quality of life made a significant contribution to the prediction of FCR. Conclusions: Post HSCT follow-up care should include an assessment and discussion regarding FCR to balance both realistic and unrealistic cancer recurrence risks. Managing FCR is one of the most ubiquitous unmet needs of survivors of haematological disease and it is important that HSCT nurses are both aware of the fear, and are equipped with knowledge on how to help patients navigate it with realistic expectations.
\end{abstract}

\footnotetext{
* Corresponding author. Graduate School of Heath, University of Technology Sydney, PO Box 123 Broadway, NSW, 2007, Australia.

E-mail address: gemma.mcerlean@uts.edu.au (G. McErlean).
} 


\section{Introduction}

Allogeneic haematopoietic stem cell transplantation (HSCT) is used worldwide to treat adults and children with a range of life-threatening malignant and non-malignant disorders including leukaemia and other haematological malignancies, immunodeficiency syndromes, haemoglobinopathies and metabolic disorders (Majhail et al., 2015). In recent years improvements in donor selection, chemo-radiotherapy conditioning and supportive care have meant that an increasing number of patients will become long-term survivors of allogeneic HSCT (Kliman et al., 2020). Many, however, experience one or more chronic health conditions post-HSCT and many live with the possibility that their original disease will recur (Barrett and Battiwalla, 2010; Mohty and Mohty, 2011). Relapse remains the most frequent cause of treatment failure and mortality, and it is particularly common in patients who have undergone HSCT for high risk haematological malignancies or relapsed disease. Primary disease is responsible for 21 and 59\% of deaths in the first 100 days, and after the first 100 days respectively post allogeneic HSCT (depending on the primary disease and donor type) (D'Souza et al., 2020). Consequently, many HSCT survivors live with the anxiety and fear associated with disease recurrence (Bacigalupo et al., 2004; Gilroy et al., 2016; Serna et al., 2003).

Fear, worry or concern relating to the possibility that cancer will come back, or progress, has been termed the Fear of Cancer Recurrence (FCR) and is estimated to affect between $22 \%$ and $87 \%$ of cancer survivors (Lebel et al., 2016). A number of psychological, medical, behavioural and socio-demographic factors have been found to predict its occurrence, including a diagnosis of anxiety or depression, young age at treatment, years since treatment, poorer quality of life (QoL), experiencing adverse effects of treatment, complementary medicine use, educational status, gender, income, occupation and ethnicity (Simard and Savard, 2009; Simard et al., 2010a). Systematic reviews of FCR have identified younger age at transplant as a key demographic determinant of FCR and have found moderate evidence for a relationship between FCR and educational status, gender, income, occupation and ethnicity (Costanzo et al., 2007; Koch et al., 2013; Sarkar et al., 2014b; Thewes et al., 2012a). Correlations between years since treatment and medical side effects and FCR have also been established (Sarkar et al., 2014b; Thewes et al., 2012a). Interestingly, despite research demonstrating strong associations between anxiety, depression and FCR, it has been found that FCR is not related to psychotropic medication use or having psychiatric treatment (Koch et al., 2013; Rabin et al., 2004; Skaali et al., 2009). Furthermore, higher FCR has been shown to be associated with complementary therapies use and health promoting behaviours, including maintaining a healthy diet, using sunscreen and attending regular health reviews (Egger et al., 2018). FCR has not been shown to be associated with behaviours that may increase the risk of cancer, including alcohol consumption or smoking (Hawkins et al., 2010).

The impact of FCR appears to be profound, with studies suggesting that it is one of the most significant psychological challenges that cancer survivors face and is one of the most frequent unmet supportive care needs (Armes et al., 2009; Beesley et al., 2013; McDowell et al., 2010; Minstrell et al., 2008). While FCR has been described in patients who have undergone chemotherapy, surgery or radiotherapy to treat solid cancers, little is known about FCR following allogeneic HSCT for haematological malignancy (Sarkar et al., 2014a). This represents a major limitation of what is known about the psychological experience of HSCT survivors as the majority of patients who undergo transplant have haematological cancers, and relapse post allogeneic HSCT is infrequently curable (Barrett and Battiwalla, 2010; D'Souza et al., 2020). As is the case with HSCT more generally, patients with cancer who undergo HSCT have generally exhausted most other treatment options and HSCT is typically undertaken because it represents the best (and often last) option for cure.

The aim of this study was to determine the prevalence of FCR in patients with a haematological malignancy surviving more than one- year post allogenic HSCT and to identify the demographic, medical and psychological factors associated with its occurrence. It is hoped that the information gleaned from this study will inform the design and delivery of health services to survivors of allogenic HSCT, and will aid in the education and support of those patients and families undergoing transplant. This is important for HSCT Nurses as they are often the first line of contact between survivors and the HSCT team.

\section{Methods}

\subsection{Patients and procedures}

This manuscript reports results from a larger cross-sectional survey study assessing the health, financial, cognitive, sexual and psychosocial experience of life post-transplant of allogeneic HSCT survivors (Brice et al., 2017; Dyer et al., 2016a, 2016b, 2018; Gifford et al., 2016). The study sample was selected from allogeneic transplant databases of all four major metropolitan hospitals in New South Wales (NSW), Australia. Participants were eligible if they were $\geq 18$ years of age and had undergone an allogeneic HSCT for a haematological malignancy between January 1, 2000 and December 31, 2012 and could read and write English. Potential participants were given the option of self-completing the survey or completing it with one of the researchers in a phone interview. All participants chose to self-complete the questionnaire. Surveys were given out in clinic, or sent in the mail, to eligible participants between October 2013-December 2013, and study close date was March 2104. All participants were given two months to return the survey before receiving a reminder phone call. After follow-up phone calls, 17 people explicitly declined and 125 did not return the survey. The study protocol was approved by the Northern Sydney Local Health District Human Research Ethics Committee (NSLHD Reference: 1207-217 M).

\subsection{Instruments}

Study participants completed a purpose designed questionnaire; the Sydney post BMT Study Survey, as well as a range of other validated instruments to explore their quality of life (QoL), psychological morbidity and health concerns post-HSCT. This include the Functional Assessment of Cancer Therapy - Bone Marrow Transplant (FACT-BMT Version 4) (Cella et al., 1993; McQuellon et al., 1997) anxiety stress and depression (The DASS 21) (Crawford and Henry, 2003; Dahm et al., 2013; Lovibond, 1996), chronic GVHD (The Chronic GVHD Activity Assessment - Patient Self Report (Form B) (Pavletic et al., 2006) and The Lee Chronic GVHD Symptom Scale) (Lee et al., 2002), The Post Traumatic Growth Inventory Score (Morris et al., 2013; Tedeschi and Calhoun, 1996) and the Fear of Recurrence Scale (Greenberg et al., 1997). These surveys are described next.

The Sydney Post-BMT Survey was devised by the research team in collaboration with health professionals involved in the care of HSCT patients, including haematologists, infectious disease physicians, HSCT nurses, HSCT psychologists, and dieticians, and discussions with transplant survivors attending HSCT clinics. It is a 402-question survey covering twenty areas including:

1. Demographics (6 questions)

2. Medical complications (36 questions)

3. Referrals, tests and assessment and time (35 questions)

4. Medications and treatments (27 questions)

5. Oral and dental health (15 questions)

6. Infections (17 questions)

7. Vaccinations (30 questions)

8. Complementary therapies (17 questions)

9. Cancer screening (37 questions)

10. Travel history (36 questions)

11. Close personal contacts (6 questions)

12. Lifestyle (10 questions) 
13. Diet/Nutrition (19 questions)

14. Occupation - Infection risk (11 questions)

15. Occupation - works status and functioning (35 questions)

16. Fertility and sexual function (41 questions)

17. Relationships (3 questions)

18. Preference for long term follow up care (8 questions)

19. Social, occupational attitudes, physical and psychological concerns (12 questions)

20. The three things that have impacted you most (1 question)

The SPBS survey used tick box responses, short answer questions and 5-point Likert scales measuring attitudes and other factors and took approximately $1 \mathrm{~h}$ to complete. It was piloted in two HSCT clinics by six HSCT survivors to check for face and content validity. No changes were required after piloting. A copy of the survey can be found in the supplementary material.

\subsubsection{Functional Assessment of Cancer Therapy - Bone Marrow Transplant (FACT-BMT version 4)}

The FACT-BMT is a validated questionnaire for measuring QoL in HSCT recipients (7). It takes three to $5 \mathrm{~min}$ to complete and combines two instruments, the FACT-G and a HSCT subscale. The FACT-G is a twenty eight-item self-report instrument that measures QoL in cancer patients (8). It consists of five subscales measuring physical, functional, social and emotional well-being and satisfaction with the doctor/patient relationship. The BMT subscale includes twelve items designed to test QoL in HSCT patients. The FACT-BMT plus the BMT subscale provides an overall QoL score. Patients rate themselves over the past seven days using five-step Likert scales with responses used to calculate overall QoL and subscale wellbeing scores.

\subsubsection{The Chronic GVHD Activity Assessment - Patient Self Report (form B)}

The Chronic GVHD Activity Assessment - Patient Self Report Form B was developed by the NIH Consensus Development Project (9). It is a ten-item questionnaire which asks patients to report on the severity and intensity (out of 10) of skin, oral, ocular and vulvovaginal symptoms as well as perceived global ratings of GVHD. It takes about $1 \mathrm{~min}$ to complete.

\subsubsection{The Lee Chronic GVHD Symptom Scale}

The Lee Chronic GVHD Symptom Scale is a thirty-item validated questionnaire for measuring symptoms of cGVHD (10). It consists of seven subscales measuring adverse effects of cGVHD on skin, eyes, mouth, lungs, nutritional status, muscles and joints, vitality and psychological functioning. Patients rate themselves over the past month using five-step Likert scales. It takes about $2 \mathrm{~min}$ to complete.

\subsubsection{The Post Traumatic Growth Inventory}

The Post Traumatic Growth Inventory is a twenty one-item questionnaire which measures post traumatic growth experiences in trauma survivors' lives (11). It is widely used to assess positive life changes following traumatic events such as cancer, HIV, rape and disasters and other crises (12). Statements including 'I developed new interests', 'I know that I can handle difficult situations' and 'I learned a great deal about how wonderful people are' expressed and the reader is asked to respond using a six-point Likert scale with responses ranging from, 'I did not experience this change' to 'I experienced this change to a very great degree as a result of my crisis'.

\subsubsection{The DASS 21}

The Depression, anxiety and stress scale (DASS 21) is a twenty oneitem self-report questionnaire designed to measure the severity of a range of symptoms common to both depression and anxiety (15). It uses a four-point Likert scale and each question is scored out of three for an overall total score out of sixty three. A higher score indicates greater

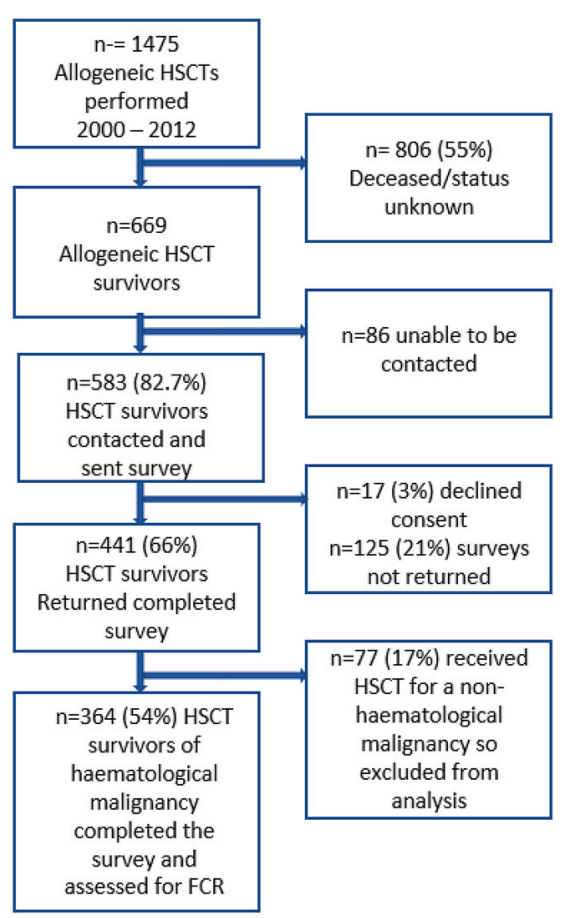

Fig. 1. Study flowchart.

severity of symptoms of anxiety or depression.

\subsubsection{The Fear of Recurrence Scale}

The Fear of Recurrence Scale was developed in the early 1990s by the authors of a study looking at.

QoL in Leukaemia patients. It consists of five questions which measure individual's thoughts surrounding recurrence of their disease. Each question is scored on a 5-point likert scale, with the total possible score range from 5 to 25 . Higher scores indicate higher FCR; a score of $\geq 4$ on any question $=$ severe FCR (Greenberg et al., 1997).

\subsubsection{Clinical HSCT variables}

Clinical HSCT variables were also collected from the transplant databases of the participating hospitals. These data together with data on malignancy type, demographic and social variables, comorbidities, secondary cancer diagnosis, specialist medical reviews, medications, post-transplant screening and lifestyle factors, were assessed for their association with FCR.

\subsection{Statistical analysis}

Categorical responses were summarised using frequencies and percentages. Parametric continuous variables were summarised using means and standard deviations, and non-parametric variables using medians and interquartile ranges. The Pearson $\chi^{2}$ test or Fishers Exact tests were used for comparative analysis of dichotomous categorical variables. Two sample comparisons of parametric and nonparametric continuous data were determined using the independent $t$-test, and Wilcoxon Rank Sum tests respectively; greater than two sample comparisons were determined using one-way analysis of Variance (ANOVA) and Kruskal Wallis tests. A two-tailed p value $<0.05$ was used as the level of statistical significance. Hierarchical regression analysis was used to assess predictors of FCR. Statistical analysis was performed using Stata software (Version 12.1).

\section{Results}

A total of 1475 allogeneic HSCT were performed in the study period 
Table 1

Demographic, social and transplant variable of study respondents.

\begin{tabular}{|c|c|}
\hline Demographic and social variables & Results ( $\mathrm{N}=$ number of responses) \\
\hline Median years since transplant (IQR; range) & 5 (IQR: 3, 8; range 1-14) \\
\hline \multicolumn{2}{|l|}{ Years since transplant, category $(\mathrm{N}=441)$} \\
\hline$<2 \mathrm{yrs}$ & $58(13.2 \%)$ \\
\hline 2 to $<6$ yrs & $204(46.3 \%)$ \\
\hline 6 to $<10 \mathrm{yrs}$ & $117(26.5 \%)$ \\
\hline $10-14$ yrs & $62(14.1 \%)$ \\
\hline Median age at survey, years (IQR; range) & 54 (IQR: 44,62; range 19-79) \\
\hline $\begin{array}{l}\text { Median age at transplant, years (IQR; } \\
\text { range) }\end{array}$ & 49 (IQR: 38,56; range 17-71) \\
\hline \multicolumn{2}{|l|}{ Age groups, years $(N=441)$} \\
\hline $19-29$ & $30(6.8 \%)$ \\
\hline $30-39$ & $49(11.1 \%)$ \\
\hline $40-49$ & $83(18.8 \%)$ \\
\hline $50-59$ & $130(29.5 \%)$ \\
\hline $60-69$ & $127(28.8 \%)$ \\
\hline$>70$ & $22(5.0 \%)$ \\
\hline \multicolumn{2}{|l|}{ Gender $(N=441)$} \\
\hline Male & $250(56.7 \%)$ \\
\hline Female & $191(43.3 \%)$ \\
\hline \multicolumn{2}{|l|}{ Culture, ethnicity $(\mathrm{N}=372)$} \\
\hline Australian/European & $323(86.8 \%)$ \\
\hline Indigenous Australian & $2(0.5 \%)$ \\
\hline Asian & $30(8.1 \%)$ \\
\hline Middle Eastern & $7(1.9 \%)$ \\
\hline Other & $10(2.7 \%)$ \\
\hline \multicolumn{2}{|l|}{ Education $(\mathrm{N}=333)$} \\
\hline Some high school & $53(15.9 \%)$ \\
\hline Completed High school & $79(23.7 \%)$ \\
\hline Trade qualifications/diploma & $47(14.1 \%)$ \\
\hline Some university & $24(7.2 \%)$ \\
\hline Completed university & $130(39.0 \%)$ \\
\hline \multicolumn{2}{|c|}{ Household income, post-transplant $(\mathrm{N}=423)$} \\
\hline Low income $\$ 20,000-39,999$ & $155(36.6 \%)$ \\
\hline Middle income $\$ 40,000-79,999$ & $123(29.1 \%)$ \\
\hline High income $\geq \$ 80,000$ & $145(34.3 \%)$ \\
\hline \multicolumn{2}{|l|}{ Demographic and social variables } \\
\hline \multicolumn{2}{|l|}{ Employment status, post-transplant $(\mathrm{N}=412)$} \\
\hline Full-time & $131(31.8 \%)$ \\
\hline Part-time & $78(18.9 \%)$ \\
\hline Homemaker & $21(5.1 \%)$ \\
\hline Casual & $26(6.3 \%)$ \\
\hline Unemployed & $20(4.8 \%)$ \\
\hline Unable to work, poor health & $56(13.6 \%)$ \\
\hline Retired & $80(19.4 \%)$ \\
\hline \multicolumn{2}{|l|}{ Residential location $(N=431)$} \\
\hline RA1 (Major city) & $311(72.2 \%)$ \\
\hline RA2 (Inner regional) & $85(19.7 \%)$ \\
\hline RA3 (Outer regional) & $31(7.2 \%)$ \\
\hline RA4 (Remote) & $4(0.9 \%)$ \\
\hline RA5 (Very remote) & 0 \\
\hline \multicolumn{2}{|l|}{ Relationship status $(\mathrm{N}=434)$} \\
\hline Single & $67(15.4 \%)$ \\
\hline Married & $311(71.7 \%)$ \\
\hline Defacto & $33(7.6 \%)$ \\
\hline Divorced & $18(4.2 \%)$ \\
\hline Separated & $5(1.1 \%)$ \\
\hline \multicolumn{2}{|l|}{ Transplant variables } \\
\hline Underlying diagnosis $\dagger(\mathrm{N}=423)$ & \\
\hline AML/ALL & $169 / 57=226(53.4 \%)$ \\
\hline CML & $21(5.0 \%)$ \\
\hline CLL & $19(4.5 \%)$ \\
\hline SAA & $16(3.8 \%)$ \\
\hline NHL & $79(18.7 \%)$ \\
\hline HL & $5(1.2 \%)$ \\
\hline MM & $14(3.3 \%)$ \\
\hline MDS/myeloproliferative disorder & $39(9.2 \%)$ \\
\hline Other (unspecified) & $4(0.9 \%)$ \\
\hline Remission status $(\mathrm{N}=405)$ & \\
\hline CR1/CR2 & $271(66.9 \%)$ \\
\hline$>\mathrm{CR} 2$ & $22(5.4 \%)$ \\
\hline Chronic Phase & $18(4.4 \%)$ \\
\hline Accelerated Phase and blast crisis & $3(0.7 \%)$ \\
\hline Refractory & $22(5.4 \%)$ \\
\hline Partial remission & $23(5.7 \%)$ \\
\hline
\end{tabular}

Table 1 (continued)

\begin{tabular}{ll}
\hline Demographic and social variables & Results $(\mathrm{N}=$ number of responses) \\
\hline Other & $46(11.4 \%)$ \\
Donor type $(\mathbf{N}=439)$ & \\
Sibling & $250(56.9 \%)$ \\
Haploidentical & $10(2.3 \%)$ \\
Matched unrelated & $158(36.0 \%)$ \\
Mismatched unrelated & $21(4.8 \%)$ \\
Stem cell source $(\mathbf{N}=441)$ & \\
Bone marrow & $48(10.9 \%)$ \\
PBSCT & $381(86.4 \%)$ \\
Cord & $12(2.7 \%)$ \\
Conditioning (N = 439) & \\
Myeloablative - proportion with TBI & $214(48.7 \%)-$ TBI $101 / 214=$ \\
& $47.2 \%$ \\
Bu/Cy & $79(36.9 \%)$ \\
Cy/TBI & $99(46.3 \%)$ \\
Bu/Flu & $28(13.1 \%)$ \\
Cy/ATGAM & $5(2.3 \%)$ \\
Cy/Flu/ATGAM & $1(0.5 \%)$ \\
Bu/Flu/Thymoglobulin/TBI & $1(0.5 \%)$ \\
Etop/TBI & $1(0.5 \%)$ \\
Reduced-intensity - proportion with TBI & $225(51.3 \%)-$ TBI 26/225=11.6\% \\
Flu/Cy & $24(10.7 \%)$ \\
Flu/Cy/TBI & $14(6.2 \%)$ \\
Flu/Mel & $98(43.6 \%)$ \\
FLAMSA & $1(0.4 \%)$ \\
Flu/BCNU/Mel/ATG & $42(18.7 \%)$ \\
Flu/TBI & $12(5.3 \%)$ \\
Other (unspecified) & $34(15.1 \%)$ \\
\hline &
\end{tabular}

across four major transplant centres in Australia. Four hundred and forty-one HSCT survivors (66\% of total eligible, $76 \%$ of those contacted) returned the completed survey. Of these, 364 patients had a pretransplant diagnosis of a haematological malignancy (Fig. 1). Respondents consisted of 250 (56.7\%) males and 191 (43.3\%) females with a median age of 54 years. A total of $86.6 \%$ identified as being of Australian/European ethnicity and $72.2 \%$ lived in a major city. Most respondents (46.3\%) were between 2 and 6 years post-transplant and $53.4 \%$ had an underlying diagnosis of acute leukaemia. Many (66.9\%) reported being in CR1/CR2 at the time of transplant, over half (56.7\%) had a sibling donor, $86.4 \%$ received peripheral blood stem cells and almost half (48.7\%) received myeloablative conditioning for their BMT, with $28.6 \%$ receiving $\mathrm{T}$-cell depletion of some form (Table 1 ).

\subsection{Prevalence of FCR}

Questions relating to FCR were completed by 355 of the 364 respondents. The mean (standard deviation) of FCR scores for 355 cancer survivors was 13.22 (4.46) (Table 1). Approximately $11 \%$ of the sample (10.98\%) lived with severe FCR and only $5 \%$ of subjects reported having no symptoms to suggest FCR. All other respondents scored in the moderate range on the FCR Scale.

\subsection{Demographic, transplant factors and FCR}

There were no significant differences in mean FCR scores by age, gender, place of residence, income or ethnicity. Similarly, mean FCR scores did not differ significantly for level of education or income group. Those in full or part time employment showed significantly lower mean FCR scores compared to those not employed $(\mathrm{p}=0.01)$. Those with an acute leukaemia diagnosis in first or second remission (a prognostic factor for cure following transplant) had FCR scores that were lower than those in later stages of remission, though this was not statistically significant. Being more years out from transplantation showed a significant negative association with FCR, such that those in the first two years post-transplant had the highest FCR scores $(\mathrm{p}<0.0001)$ (Table 2$)$. 
Table 2

Demographic, social, transplant variables and their associations with fear of cancer recurrence (FCR) post HSCT.

\begin{tabular}{|c|c|c|}
\hline Variables & FCR Score Mean (sd) & $P$ value \\
\hline \multicolumn{3}{|l|}{ Demographic } \\
\hline \multicolumn{3}{|l|}{ Gender } \\
\hline Male & $13.46(4.40)$ & 0.23 \\
\hline Female & $12.89(4.52)$ & \\
\hline \multicolumn{3}{|l|}{ Age (years) } \\
\hline$<54(\mathrm{n}=179)$ & $13.08(4.64)$ & 0.55 \\
\hline$\geq 54(n=176)$ & $13.36(4.27)$ & \\
\hline \multicolumn{3}{|l|}{ Postcode } \\
\hline City-Metro $(\mathrm{n}=252)$ & $13.25(4.61)$ & 0.74 \\
\hline Regional or remote $(n=95)$ & $13.07(4.12)$ & \\
\hline \multicolumn{3}{|l|}{ Ethnicity } \\
\hline Caucasian/European $(n=261)$ & $12.99(4.52)$ & 0.62 \\
\hline Other $(n=42)$ & $13.36(4.52)$ & \\
\hline \multicolumn{3}{|l|}{ Socioeconomic } \\
\hline \multicolumn{3}{|l|}{ Education } \\
\hline Some high school $(n=40)$ & $13.32(4.18)$ & 0.98 \\
\hline Completed High school $(n=60)$ & $13.08(4.56)$ & \\
\hline Trade/diploma $(\mathrm{n}=41)$ & $12.90(4.01)$ & \\
\hline Some university $(\mathrm{n}=17)$ & $13.00(4.17)$ & \\
\hline Completed university $(\mathrm{n}=112)$ & $13.34(4.73)$ & \\
\hline University Education $(\mathrm{n}=129)$ & $13.29(4.64)$ & 0.72 \\
\hline No University Education $(\mathrm{n}=141)$ & $13.10(4.27)$ & \\
\hline \multicolumn{3}{|l|}{ Post-transplant income } \\
\hline Low income $(\mathrm{n}=123)^{\wedge \wedge}$ & $13.54(4.37)$ & 0.29 \\
\hline Middle-High income $(\mathrm{n}=217)$ & $13.00(4.57)$ & \\
\hline \multicolumn{3}{|l|}{ Occupational status } \\
\hline Full/Part time $(\mathrm{n}=170)$ & $12.66(4.45)$ & 0.01 \\
\hline Other $*(n=159)$ & $13.89(4.39)$ & \\
\hline \multicolumn{3}{|l|}{ Marital status } \\
\hline Married or defacto $(n=283)$ & $13.45(4.38)$ & 0.11 \\
\hline Other $* *(n=67)$ & $12.48(4.76)$ & \\
\hline \multicolumn{3}{|l|}{ Transplant factors } \\
\hline \multicolumn{3}{|l|}{ Pretransplant Cancer Diagnosis } \\
\hline Acute Leukaemia $(\mathrm{n}=223)$ & $13.03(4.74)$ & 0.31 \\
\hline Other $(n=132)$ & $13.53(4.28)$ & \\
\hline \multicolumn{3}{|c|}{ Pretransplant remission status in those with AL } \\
\hline $\mathrm{CR} 1 / 2(\mathrm{n}=208)$ & $12.89(4.25)$ & 0.06 \\
\hline Other $(\mathrm{n}=15)$ & $15.00(4.31)$ & \\
\hline \multicolumn{3}{|l|}{ Years since transplant } \\
\hline$<2$ yrs $(\mathrm{n}=51)$ & $15.37(4.49)$ & $<0.0001$ \\
\hline $2<6$ yrs $(n=160)$ & $13.67(4.22)$ & \\
\hline $6<10$ yrs $(n=94)$ & $11.84(4.35)$ & \\
\hline$\geq 10 y r s(n=50)$ & $12.16(4.34)$ & \\
\hline \multicolumn{3}{|l|}{ Conditioning } \\
\hline Myeloablative $(\mathrm{n}=175)$ & $13.11(4.60)$ & 0.65 \\
\hline Reduced Intensity $(\mathrm{n}=178)$ & $13.33(4.35)$ & \\
\hline
\end{tabular}

\subsection{Medical co-morbidity and FCR}

Medical conditions diagnosed since transplantation that showed an association with increased mean FCR included skin cancer $(\mathrm{p}=0.01)$, depression $(p=0.01)$ and anxiety $(p=0.003)$ (see Table 3$)$. Higher mean FCR scores were reported in those taking antidepressants $(\mathrm{p}=$ $0.04)$, anxiolytics $(p=0.02)$ and sedatives $(p=0.01)$. Mean FCR scores were significantly higher for those referred to psychiatrists $(\mathrm{p}=0.003)$.

\subsection{Routine cancer screening and FCR}

Potential associations between FCR and the uptake of routinely recommended screening for post-transplant secondary malignancies were explored. No mean differences in FCR were seen in those who did or did not attend skin checks, bowel screening, mammography (females) or prostate checks (males) including those who underwent these screening procedures either early or late post-transplant. The only significant difference in mean FCR was in women who did not report at least one PAP smear since transplant. Those HSCT survivors who had not attended a PAP smear post HSCT reported significantly higher mean FCR scores $(p=0.003)$. This was significant for women who reported no Pap screening early post-transplant $(\mathrm{p}=0.004)$ (Table 4$)$.
Table 3

Post transplant diagnosis, psychotropic medication and mental health referrals and their association with fear of cancer recurrence (FCR).

\begin{tabular}{|c|c|c|}
\hline Clinical factors & FCR score Mean (sd) & $P$ value \\
\hline \multicolumn{3}{|l|}{ Thyroid Disease } \\
\hline Yes $(n=15)$ & $13.60(4.92)$ & 0.72 \\
\hline No $(\mathrm{n}=298)$ & $13.18(4.34)$ & \\
\hline \multicolumn{3}{|l|}{ Bone Disease } \\
\hline Yes $(n=104)$ & $13.18(4.35)$ & 0.99 \\
\hline No $(n=219)$ & $13.22(4.49)$ & \\
\hline \multicolumn{3}{|l|}{ CVS risk factors } \\
\hline Yes $(n=148)$ & $13.04(4.28)$ & 0.81 \\
\hline $\operatorname{No}(\mathrm{n}=181)$ & $13.16(4.52)$ & \\
\hline \multicolumn{3}{|l|}{ Cataracts } \\
\hline Yes $(n=100)$ & $13.09(4.11)$ & 0.88 \\
\hline No $(n=228)$ & $13.17(4.51)$ & \\
\hline \multicolumn{3}{|l|}{ Iron Overload } \\
\hline Yes $(n=100)$ & $12.94(4.08)$ & 0.66 \\
\hline No $(n=223)$ & $13.17(4.50)$ & \\
\hline \multicolumn{3}{|l|}{ Recurrent colds } \\
\hline Yes $(n=80)$ & $13.44(4.35)$ & 0.43 \\
\hline No $(n=242)$ & $12.99(4.36)$ & \\
\hline \multicolumn{3}{|l|}{ Mouth Cancer } \\
\hline Yes $(n=6)$ & $16.33(3.50)$ & 0.07 \\
\hline No $(n=308)$ & $13.06(4.41)$ & \\
\hline \multicolumn{3}{|l|}{ Skin cancer } \\
\hline Yes $(n=74)$ & $14.25(4.37)$ & 0.01 \\
\hline No $(n=251)$ & $12.82(4.49)$ & \\
\hline \multicolumn{3}{|l|}{ Other Cancer } \\
\hline Yes $(n=13)$ & $15.07(4.57)$ & 0.13 \\
\hline No $(\mathrm{n}=342)$ & $13.15(4.44)$ & \\
\hline \multicolumn{3}{|l|}{ Depression } \\
\hline Yes $(n=74)$ & $14.54(4.64)$ & 0.006 \\
\hline No $(n=250)$ & $12.91(4.38)$ & \\
\hline \multicolumn{3}{|l|}{ Anxiety } \\
\hline Yes $(n=66)$ & $14.65(4.38)$ & 0.003 \\
\hline No $(\mathrm{n}=257)$ & $12.86(4.40)$ & \\
\hline \multicolumn{3}{|l|}{ cGVHD } \\
\hline Yes $(n=244)$ & $13.40(4.43)$ & 0.13 \\
\hline No $(n=104)$ & $12.61(4.47)$ & \\
\hline \multicolumn{3}{|l|}{ Medications } \\
\hline \multicolumn{3}{|l|}{ Antidepressant } \\
\hline Yes $(n=43)$ & $14.49(4.82)$ & 0.04 \\
\hline No $(\mathrm{n}=312)$ & $13.04(4.39)$ & \\
\hline \multicolumn{3}{|l|}{ Anxiolytic } \\
\hline Yes $(n=23)$ & $15.35(4.79)$ & 0.02 \\
\hline No $(\mathrm{n}=332)$ & $13.07(4.40)$ & \\
\hline \multicolumn{3}{|c|}{ Sedation/sleeping tablets } \\
\hline Yes $(n=39)$ & $15.02(4.60)$ & 0.007 \\
\hline No $(\mathrm{n}=317)$ & $12.98(4.40)$ & \\
\hline Mental health referral & FCR Score Mean (sd) & $P$ value \\
\hline \multicolumn{3}{|l|}{ Psychologist } \\
\hline Yes $(n=65)$ & $13.89(4.44)$ & 0.20 \\
\hline & $13.10(4.47)$ & \\
\hline \multicolumn{3}{|l|}{ Psychiatrist } \\
\hline Yes $(n=26)$ & $15.77(5.13)$ & 0.003 \\
\hline & $13.05(4.39)$ & \\
\hline \multicolumn{3}{|l|}{ Social Worker } \\
\hline Yes $(n=44)$ & $14.29(4.39)$ & 0.09 \\
\hline No $(n=289)$ & $13.07(4.42)$ & \\
\hline
\end{tabular}

\subsection{Lifestyle \& complementary therapies and FCR}

No significant correlation was found between FCR and different health behaviours and lifestyle choices including cigarette smoking, alcohol consumption, exercise or the routine use of sunscreen, and those who elected to use a range of complementary therapies. (Table 5).

\subsection{Psychological variables, regression analysis and FCR}

Zero-order correlations were calculated for all variables included in the hierarchical multiple regression (Table 6). Full- or part-time employment, prescription of psychopharmacological medications and psychological distress all showed a significant positive correlation with 
Table 4

Screening for secondary malignancies and fear of cancer recurrence (FCR) post HSCT.

\begin{tabular}{|c|c|c|c|c|c|c|c|c|}
\hline Screening & $\begin{array}{l}\text { FCR Score Mean } \\
\text { (sd) }\end{array}$ & $P$ value & $\begin{array}{l}\text { Early screening }<2 \\
\text { yrs }\end{array}$ & $\begin{array}{l}\text { FCR Mean }(\mathrm{sd})< \\
2 \text { yrs }\end{array}$ & $\begin{array}{l}\text { P value } \\
<2 \text { yrs }\end{array}$ & $\begin{array}{l}\text { Late screening } \geq 2 \\
\text { yrs }\end{array}$ & $\begin{array}{l}\text { FCR Mean (sd) } \\
\geq 2 \text { yrs }\end{array}$ & $\begin{array}{l}\mathrm{P} \\
\text { value } \geq 2 \mathrm{yrs}\end{array}$ \\
\hline \multicolumn{9}{|l|}{ Skin checks } \\
\hline Yes $(n=174)$ & $12.98(4.60)$ & 0.24 & Yes $(n=18)$ & $15.78(5.00)$ & 0.69 & Yes $(n=156)$ & $12.66(4.45)$ & 0.32 \\
\hline No $(n=177)$ & $13.54(4.32)$ & & No $(\mathrm{n}=32)$ & $15.25(4.29)$ & & No $(n=145)$ & $13.16(4.24)$ & \\
\hline \multicolumn{9}{|l|}{ Bowel cancer } \\
\hline Yes $(n=116)$ & $12.94(3.95)$ & 0.35 & Yes $(n=13)$ & $15.23(3.10)$ & 0.88 & Yes $(n=103)$ & $12.65(3.88)$ & 0.47 \\
\hline No $(\mathrm{n}=232)$ & $13.42(4.71)$ & & No $(\mathrm{n}=37)$ & $15.46(4.79)$ & & No $(n=195)$ & $13.03(4.60)$ & \\
\hline \multicolumn{9}{|c|}{ Pap smears (Females) } \\
\hline Yes $(n=95)$ & $12.05(3.37)$ & 0.003 & Yes $(\mathrm{n}=7)$ & $13.00(4.24)$ & 0.04 & Yes $(\mathrm{n}=88)$ & $11.98(4.39)$ & 0.07 \\
\hline No $(n=53)$ & $14.30(4.41)$ & & No $(n=11)$ & $17.45(3.96)$ & & No $(n=42)$ & 13.48 (4.19) & \\
\hline \multicolumn{9}{|c|}{ Mammogram (Females) } \\
\hline Yes $(n=77)$ & $12.48(4.00)$ & 0.47 & Yes $(n=6)$ & $16.00(6.26)$ & 0.63 & Yes $(\mathrm{n}=71)$ & $12.18(3.66)$ & 0.49 \\
\hline No $(n=70)$ & $13.01(4.94)$ & & No $(n=10)$ & $14.80(3.58)$ & & No $(n=60)$ & $12.72(5.09)$ & \\
\hline \multicolumn{9}{|c|}{ Prostate check (Males) } \\
\hline Yes $(n=71)$ & $13.84(4.13)$ & 0.38 & Yes $(\mathrm{n}=12)$ & $15.75(4.92)$ & 0.60 & Yes $(\mathrm{n}=59)$ & $13.46(3.89)$ & 0.49 \\
\hline No $(\mathrm{n}=129)$ & $13.26(4.60)$ & & No $(n=20)$ & $14.85(4.50)$ & & No $(\mathrm{n}=109)$ & $12.97(4.58)$ & \\
\hline
\end{tabular}

Table 5

Lifestyle choices, including complementary therapy use, and fear of cancer recurrence in long-term follow-up post HSCT.

\begin{tabular}{|c|c|c|}
\hline Life style factor & FCR Score Mean (sd) & $P$ value \\
\hline \multicolumn{3}{|l|}{ Smoking } \\
\hline Yes $(n=28)$ & $12.68(4.01)$ & 0.50 \\
\hline No $(\mathrm{n}=327)$ & $13.27(4.50)$ & \\
\hline \multicolumn{3}{|l|}{ Drinking alcohol } \\
\hline Yes $(n=227)$ & $12.92(4.44)$ & 0.10 \\
\hline No $(n=128)$ & $13.74(4.46)$ & \\
\hline \multicolumn{3}{|l|}{ Heavy alcohol* } \\
\hline Yes $(n=27)$ & $12.07(3.33)$ & 0.36 \\
\hline No $(\mathrm{n}=192)$ & $12.91(4.56)$ & \\
\hline \multicolumn{3}{|l|}{ Exercise, sport } \\
\hline Yes $(n=244)$ & $12.93(4.35)$ & 0.06 \\
\hline No $(\mathrm{n}=109)$ & $13.90(4.59)$ & \\
\hline \multicolumn{3}{|c|}{ Of those who Regular Exercise (at least 3x/week) } \\
\hline Yes (161) & $12.82(4.41)$ & 0.68 \\
\hline No (77) & $13.08(4.33)$ & \\
\hline \multicolumn{3}{|l|}{ Routine use of sunscreen } \\
\hline Yes $(n=268)$ & $13.41(4.37)$ & 0.21 \\
\hline No $(\mathrm{n}=78)$ & $12.70(4.57)$ & \\
\hline Complementary therapy & FCR Score Mean (sd) & $P$ value \\
\hline \multicolumn{3}{|c|}{ Nutrition \&dietary approaches } \\
\hline Yes $(n=45)$ & $12.18(4.83)$ & 0.10 \\
\hline No $(\mathrm{n}=303)$ & $13.35(4.41)$ & \\
\hline \multicolumn{3}{|l|}{ Herbal supplements } \\
\hline Yes $(n=49)$ & $12.96(4.86)$ & 0.65 \\
\hline No $(\mathrm{n}=295)$ & $13.27(4.37)$ & \\
\hline \multicolumn{3}{|l|}{ Vitamin therapies } \\
\hline Yes $(n=101)$ & 13.27 (4.59) & 0.97 \\
\hline No $(n=239)$ & $13.28(4.44)$ & \\
\hline \multicolumn{3}{|l|}{ Mind-body therapies } \\
\hline Yes $(n=57)$ & $12.81(4.52)$ & 0.42 \\
\hline $\operatorname{No}(n=286)$ & $13.33(4.46)$ & \\
\hline \multicolumn{3}{|c|}{ Manipulative and body based therapies } \\
\hline Yes $(\mathrm{n}=91)$ & $12.93(4.52)$ & 0.42 \\
\hline No $(n=254)$ & $13.37(4.44)$ & \\
\hline \multicolumn{3}{|c|}{ Traditional whole medicine systems } \\
\hline Yes $(n=15)$ & $12.53(4.60)$ & 0.55 \\
\hline No $(\mathrm{n}=327)$ & $13.24(4.45)$ & \\
\hline \multicolumn{3}{|l|}{ Energy medicine } \\
\hline Yes $(n=11)$ & $12.18(5.45)$ & 0.44 \\
\hline No $(\mathrm{n}=332)$ & $13.24(4.41)$ & \\
\hline \multicolumn{3}{|l|}{ Homeopathy } \\
\hline Yes $(n=11)$ & $11.90(5.27)$ & 0.32 \\
\hline $\operatorname{No}(\mathrm{n}=329)$ & $13.27(4.43)$ & \\
\hline
\end{tabular}

$* *$ We also analysed the association between heavy alcohol use and FCR. Those with Heavy alcohol use did not demonstrate a significant association with FCR. The mean FCR scores in those with heavy alcohol use was higher than those reporting moderate/low alcohol consumption though this was not significant. The definition of heavy alcohol use was more than 2 standard drinks of alcohol per day (or $>14$ /week).
Table 6

Intercorrelations between hierarchical regression variables.

\begin{tabular}{|c|c|c|c|c|c|c|}
\hline & 1 & 2 & 3 & 4 & 5 & 6 \\
\hline 1. FCR & - & & & & & \\
\hline 2. Age & .08 & - & & & & \\
\hline $\begin{array}{l}\text { 3. Employed full or } \\
\text { part time }\end{array}$ & $.16^{* *}$ & $.39 * * *$ & - & & & \\
\hline $\begin{array}{l}\text { 4. Psychotic } \\
\text { medication }\end{array}$ & $.18^{* * * *}$ & -.03 & .11 & - & & \\
\hline $\begin{array}{l}\text { 5. Years since } \\
\text { transplant }\end{array}$ & $-.23^{* * * *}$ & .04 & $-.15^{* *}$ & -.05 & - & \\
\hline $\begin{array}{l}\text { 6. Depression, } \\
\text { Anxiety, Stress } \\
\text { (DASS 21) }\end{array}$ & $.36^{* * * *}$ & -.02 & .10 & $.26^{* * *}$ & .04 & - \\
\hline $\begin{array}{l}\text { 7. Quality of Life } \\
\text { (Total FACT) }\end{array}$ & $-.43^{* * *}$ & -.001 & $-.28 * * *$ & $-.30 * * *$ & .04 & $-.66^{* * *}$ \\
\hline
\end{tabular}

Note. ${ }^{*} p<0.05,{ }^{* *} p<0.01,{ }^{* * *} p<0.001$.

FCR while the number of years since transplant and QoL demonstrated a significant negative correlation with FCR. The two key variables of interest, psychological distress (depression, anxiety and stress), and QoL were significantly negatively correlated with one another (Table 6).

Hierarchical regression was used to test the degree to which age, fullor part-time employment, psychopharmacological medication use (anxiolytics, antidepressants and sedatives), years since transplant and current levels of psychological distress and QoL can predict FCR. In the first step, age, full- or part-time employment, currently taking psychotic medication and years since transplant were entered and significantly predicted FCR, $F(4,296)=7.94, p<0.001$. Only current taking of psychotic medication and the number of years since transplant made a significant contribution to the prediction of FCR, explaining $3.6 \%$ and $3.2 \%$ of the variance, respectively. The psychological distress score and the QoL total scores were entered in the second step and $F$ change indicated a significant improvement in prediction over the use of the socio-demographic variables alone, $F(6,294)=17.01, p<0.001$. Psychological distress and QoL made a significant contribution to the prediction of FCR explaining $1.1 \%$ and $5.4 \%$, of the variance in FCR. After controlling for socio-demographic variables, higher levels of psychological distress and lower QoL significantly predicted higher levels of FCR. These results also show that the number of years since transplant significantly predicted lower FCR and that age was positively related to increased FCR (Table 7).

\section{Discussion}

While numerous studies of cancer survivors have described high rates of FCR, little is known about its occurrence in survivors of allogeneic transplant for haematological malignancy. The results of this 
Table 7

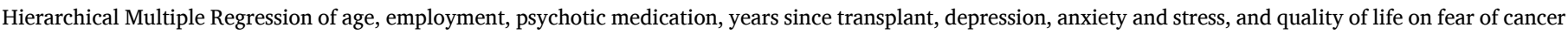
recurrence (FCR).

\begin{tabular}{|c|c|c|c|c|c|c|c|c|}
\hline \multirow[t]{2}{*}{ Variable } & \multicolumn{4}{|l|}{ Model 1} & \multicolumn{4}{|l|}{ Model 2} \\
\hline & $b$ & $S E$ & $s r^{2}$ & $95 \% C I$ & $b$ & $S E$ & $s r^{2}$ & $95 \% C I$ \\
\hline Age & .03 & .02 & .003 & $-0.01,0.07$ & $.05^{*}$ & .02 & .013 & $0.01,0.09$ \\
\hline Employed & .11 & .12 & .004 & $-0.12,0.34$ & -.08 & .11 & .002 & $-0.30,0.13$ \\
\hline Psychotic medication & $2.33^{* * *}$ & .63 & .036 & $1.09,3.57$ & 0.99 & .60 & .007 & $-0.18,2.17$ \\
\hline Years since transplant & $-0.22^{* *}$ & .07 & .032 & $-0.37,-0.08$ & $-0.25^{* * *}$ & .07 & .035 & $-0.38,-0.12$ \\
\hline Depression, Anxiety, Stress (DASS 21) & & & & & $.04^{* *}$ & .01 & .042 & $0.01,0.06$ \\
\hline Quality of Life (Total FACT) & & & & & $-.06 * * *$ & .01 & .019 & $-0.09,-0.03$ \\
\hline$\Delta R^{2}$ & & & & & $.16^{* * *}$ & & & \\
\hline$R^{2}$ & .10 & & & & .26 & & & \\
\hline
\end{tabular}

Note. ${ }^{*} p<0.05,{ }^{* *} p<0.01,{ }^{* * *} p<0.001$.

study, which is the largest of its kind, make clear that many long-term survivors of allogeneic HSCT live with FCR; however, FCR diminishes as the time since diagnosis and treatment increases. This is an important finding for HSCT nurses, who often provide care for HSCT recipients for many years after transplant. This data is consistent with the results of previous cancer survivor studies (Deimling et al., 2006; Mehta et al., 2003; Polinsky, 1994; Vickberg, 2003).

Our results suggest that psychological distress (depression, anxiety and stress), mental health professional referral, pharmacological intervention and lower QoL scores are all correlated with higher FCR scores. Whether these cause FCR or result from it is unclear. Researchers posit a bidirectional relationship between FCR and emotional distress, with psychological distress (anxiety, depression, stress) perpetuating FCR and FCR driving distress (Black and White, 2005). In this regard we found higher mean FCR scores in those taking antidepressants, anxiolytics and sedatives, in those referred to psychiatrists and, to a lesser extent, in those referred to a psychologist or social worker. Perhaps unsurprisingly, and consistent with previous research, we found that QoL (as measured by the FACT-BMT) was also associated with FCR, with lower FCR in survivors reporting a higher QoL (Mehnert et al., 2013; Polinsky, 1994; Simard et al., 2010; Thewes et al., 2012b).

Given both the potential for relapse post HSCT and the higher incidence of secondary malignancies in long-term survivors of HSCT we explored the associations between FCR, cancer screening adherence and relevant health behaviours in HSCT survivors. This is a complex issue as higher levels of FCR has been found to be positively associated with both hyper-vigilance and avoidance type behaviours with respect to health screening (Simard et al., 2010). In this study, no mean differences in FCR were found in those who had undergone several health screenings with the exception of women who did not report at least one PAP smear since transplant. This group of women were found to have a significantly higher mean FCR score. The paradoxical nature of the relationship between FCR and taking steps to diagnose it makes interpretation of this finding very difficult as while avoidance of screening may ameliorate psychological distress in the short term, in the long term, it may predict higher levels of FCR(Stanton et al., 2002). FCR did not appear to be associated with healthy lifestyle choices, exercise or the use of complementary medicine. Importantly, the development of skin cancer post-HSCT was associated with a higher FCR, suggesting that a (new) cancer diagnosis post HSCT heightened survivor's sensitivity to their underlying treatment related diagnosis and their FCR.

In the regression analysis, younger age was negatively related to FCR. This finding is consistent with studies of other cancer populations that have reported a link between younger age and vulnerability to FCR (Simard and Savard, 2009). Exactly why younger survivors may be more likely to experience a FCR is unclear but may result from awareness of the greater 'lifetime' risk of cancer post-HSCT including the psychological and existential impact of a cancer diagnosis at a young age, and the greater family, financial and employment disruptions associated with cancer and HSCT in younger people (Gilroy et al., 2016; van de Wal et al., 2016).
Intriguingly, while the majority of studies on FCR in cancer survivors have reported no relationship between FCR and employment, our results reveal a significant association between unemployment and higher FCR (Sarkar et al., 2014b). While we are unable to determine whether employment is causative in any way, in theory at least, returning to work or gaining work post- HSCT arguably may assist survivors regain a sense of meaning and normalcy, improve psychological well-being and distract survivors from thoughts associated with FCR (Peteet, 2000; Rasmussen and Elverdam, 2008).

\section{Limitations}

There are a number of limitations to this study that suggest caution in interpreting the results and generalising them to other populations or settings. First, because this is a cross sectional study it is not possible to ascertain the temporal association between FCR and other dynamic variables such as depression, anxiety and stress. Our study also reports data from survivors of allogeneic HSCT performed in one state in Australia, which may impact the generalizability of the results. Further, it is possible that some HSCT survivors may cope with FCR by engaging in avoidance, meaning that our results may underrepresent the incidence of FCR because some of those most affected chose not to complete and return their survey. Finally, as we reported data from a single timepoint in the lives of HSCT survivors, it is possible that for some, their current life experiences negated, or heightened, their experience of FCR.

\section{Conclusion}

The sequelae of allogeneic HSCT for haematological malignancies and the lingering threats of FCR can impair the QoL of HSCT survivors and is associated with significant adverse psychological impacts. For many patients with haematological malignancies who have undergone HSCT, returning to a fulfilling life following transplant relies, in part, on the patient's ability to effectively manage FCR. Given the prevalence and impact of FCR in HSCT survivors and the lack of attention historically given to it by HSCT services and health professionals, we suggest that education and support programs pre- and post-allogeneic HSCT should provide information about the incidence, predictors and potential impact of FCR and the strategies that may be used to manage it. We believe HSCT nurses are best placed to assess for and provide this kind of service. And, just as importantly, post- HSCT follow-up should also include routine assessment of FCR in survivors so that health professionals can advise them about the realistic likelihood of relapse, support them in dealing with FCR and encourage adherence with postHSCT care, including screening for recurrence and secondary cancers. We believe this data is pivotal to HSCT nurses, as they are best placed to assess, and provide advice and support for HSCT survivors experiencing FCR. 


\section{CRediT authorship contribution statement}

Lisa Brice: Conceptualization, Methodology, Investigation, Writing original draft, Writing - review \& editing. Gemma McErlean: Conceptualization, Methodology, Investigation, Writing - original draft, Writing - review \& editing, Project administration, Funding acquisition. Caroline Donovan: Formal analysis, Writing - original draft, Writing - review \& editing. Caley Tapp: Formal analysis, Writing - original draft, Writing - review \& editing. Nicole Gilroy: Conceptualization, Methodology, Investigation, Formal analysis, Writing - original draft, Writing - review \& editing. Masura Kabir: Formal analysis, Writing - original draft, Writing - review \& editing. Matt Greenwood: Investigation, Writing - original draft, Writing - review \& editing. Stephen R. Larsen: Investigation, Writing - original draft, Writing - review \& editing. John Moore: Investigation, Writing - original draft, Writing - review \& editing. David Gottlieb: Investigation, Writing - original draft, Writing review \& editing. Mark Hertzberg: Investigation, Writing - original draft, Writing - review \& editing. Louisa Brown: Investigation, Writing original draft, Writing - review \& editing. Megan Hogg: Investigation, Writing - original draft, Writing - review \& editing. Gillian Huang: Investigation, Writing - original draft, Writing - review \& editing. Jeff Tan: Investigation, Writing - original draft, Writing - review \& editing. Christopher Ward: Investigation, Writing - original draft, Writing review \& editing, Supervision. Ian Kerridge: Conceptualization, Methodology, Investigation, Writing - original draft, Writing - review \& editing, Funding acquisition, Supervision.

\section{Declaration of competing interest}

The authors declare no conflicts of interest.

\section{Acknowledgements}

This research was funded by the New South Wales Agency for Clinical Innovation, Blood and Marrow Transplant Network and supported by the Northern Blood Research Centre in Sydney Australia.

\section{Appendix A. Supplementary data}

Supplementary data to this article can be found online at https://doi. org/10.1016/j.ejon.2020.101845.

\section{References}

Armes, J., Crowe, M., Colbourne, L., Morgan, H., Murrells, T., Oakley, C., Palmer, N., Ream, E., Young, A., Richardson, A., 2009. Patients' supportive care needs beyond the end of cancer treatment: a prospective, longitudinal survey. J. Clin. Oncol. 27, 6172-6179.

Bacigalupo, A., Sormani, M.P., Lamparelli, T., Gualandi, F., Occhini, D., Bregante, S., Raiola, A.M., di Grazia, C., Dominietto, A., Tedone, E., Piaggio, G., Podesta, M., Bruno, B., Oneto, R., Lombardi, A., Frassoni, F., Rolla, D., Rollandi, G., Viscoli, C., Ferro, C., Garbarino, L., Van Lint, M.T., 2004. Reducing transplant-related mortality after allogeneic hematopoietic stem cell transplantation. Haematologica 89, 1238-1247.

Barrett, A.J., Battiwalla, M., 2010. Relapse after allogeneic stem cell transplantation. Expet Rev. Hematol. 3, 429-441.

Beesley, V.L., Price, M.A., Webb, P.M., O’Rourke, P., Marquart, L., Butow, P.N., 2013. Changes in supportive care needs after first-line treatment for ovarian cancer: identifying care priorities and risk factors for future unmet needs. Psycho Oncol. 22, 1565-1571.

Black, E.K., White, C.A., 2005. Fear of recurrence, sense of coherence and posttraumatic stress disorder in haematological cancer survivors. Psycho Oncol. 14, 510-515.

Brice, L., Gilroy, N., Dyer, G., Kabir, M., Greenwood, M., Larsen, S., Moore, J., Kwan, J., Hertzberg, M., Brown, L., Hogg, M., Huang, G., Tan, J., Ward, C., Gottlieb, D. Kerridge, I., 2017. Haematopoietic stem cell transplantation survivorship and quality of life: is it a small world after all? Support. Care Canc. 25, 421-427.

Cella, D., Tulsky, D., Gray, G., Sarafian, B., Linn, E., Bonomi, A., Silberman, M., Yellen, S., Winicour, P., J, B., 1993. The Functional Assessment of Cancer Therapy scale: development and validation of the general measure. J. Clin. Oncol. 11, 570-579.
Costanzo, E.S., Lutgendorf, S.K., Mattes, M.L., Trehan, S., Robinson, C.B., Tewfik, F., Roman, S.L., 2007. Adjusting to life after treatment: distress and quality of life following treatment for breast cancer. Br. J. Canc. 97, 1625-1631.

Crawford, J.R., Henry, J.D., 2003. The depression anxiety stress scales (DASS): normative data and latent structure in a large non-clinical sample. Br. J. Clin. Psychol. 42, $111-131$.

D'Souza, A., Fretham, C., Lee, S.J., Arora, M., Brunner, J., Chhabra, S., Devine, S., Eapen, M., Hamadani, M., Hari, P., Pasquini, M.C., Perez, W., Phelan, R.A., Riches, M.L., Rizzo, J.D., Saber, W., Shaw, B.E., Spellman, S.R., Steinert, P., Weisdorf, D.J., Horowitz, M.M., 2020 Aug. Current Use of and Trends in Hematopoietic Cell Transplantation in the United States. Biol Blood Marrow Transplant 26 (8), e177-e182. https://doi.org/10.1016/j.bbmt.2020.04.013. Epub 2020 May 11. PMID: 32438042; PMCID: PMC7404814.

Dahm, J., Wong, D., Ponsford, J., 2013. Validity of the depression anxiety stress scales in assessing depression and anxiety following traumatic brain injury. J. Affect. Disord. 151, 392-396.

Deimling, G.T., Bowman, K.F., Sterns, S., Wagner, L.J., Kahana, B., 2006. Cancer-related health worries and psychological distress among older adult, long-term cancer survivors. Psycho Oncol. 15, 306-320.

Dyer, G., Brice, L., Gilroy, N., Kabir, M., Hertzberg, M., Greenwood, M., Larsen, S.R., Moore, J., Gottlieb, D., Huang, G., Hogg, M., Brown, L., Tan, J., Ward, C., Kerridge, I., 2018. Changes to work status and household income of long-term allogeneic blood and marrow transplant survivors in New South Wales, Australia. Bone Marrow Transplant. 53, 926-931.

Dyer, G., Gilroy, N., Bradford, J., Brice, L., Kabir, M., Greenwood, M., Larsen, S.R., Moore, J., Hertzberg, M., Kwan, J., Brown, L., Hogg, M., Huang, G., Tan, J., Ward, C., Kerridge, I., 2016a. A survey of fertility and sexual health following allogeneic haematopoietic stem cell transplantation in New South Wales, Australia. Br. J. Haematol. 172, 592-601.

Dyer, G., Gilroy, N., Brown, L., Hogg, M., Brice, L., Kabir, M., Greenwood, M., Larsen, S. R., Moore, J., Hertzberg, M., Kwan, J., Huang, G., Tan, J., Ward, C., Kerridge, I., 2016b. What they want: inclusion of blood and marrow transplantation survivor preference in the development of models of care for long-term health in Sydney, Australia. Biol. Blood Marrow Transplant. 22, 731-743.

Egger, S., Hughes, S., Smith, D.P., Chambers, S., Kahn, C., Moxey, A., O'Connell, D.L., 2018. Factors associated with the use of complementary and alternative medicines for prostate cancer by long-term survivors. Plos One 13, e0193686.

Gifford, G., Gilroy, N., Dyer, G., Brice, L., Kabir, M., Greenwood, M., Larsen, S., Moore, J., Gottlieb, D., Hertzberg, M., Kwan, J., Huang, G., Tan, J., Brown, L., Hogg, M., Ward, C., Kerridge, I., 2016. The experience of survival following allogeneic haematopoietic stem cell transplantation in New South Wales, Australia. Bone Marrow Transplant. 51, 1361-1368.

Gilroy, N., Dyer, G., Kerridge, I., Brice, L., Kabir, M., 2016. NSW Agency for Clinical Innovation, the Sydney Post-bone Marrow Transplant Survey Report. SHPN (ACI) ISBN 978-1-76000-492-7.

Greenberg, D.B., Kornblith, A.B., Herndon, J.E., Zuckerman, E., Schiffer, C.A., Weiss, R. B., Mayer, R.J., Wolchok, S.M., Holland, J.C., 1997. Quality of life for adult leukemia survivors treated on clinical trials of cancer and leukemia Group B during the period 1971-1988: predictors for later psychologic distress. Cancer 80, 1936-1944.

Hawkins, N.A., Smith, T., Zhao, L., Rodriguez, J., Berkowitz, Z., Stein, K.D., 2010 Health-related behavior change after cancer: results of the American cancer society's studies of cancer survivors (SCS). J Cancer Surviv 4, 20-32.

Kliman, D., Nivison-Smith, I., Gottlieb, D., Hamad, N., Kerridge, I., Purtill, D., Szer, J., Ma, D., 2020. Hematopoietic stem cell transplant recipients surviving at least 2 years from transplant have survival rates approaching population levels in the modern era of transplantation. Biol. Blood Marrow Transplant. 26, 1711-1718.

Koch, L., Jansen, L., Brenner, H., Arndt, V., 2013. Fear of recurrence and disease progression in long-term $(>/=5$ years) cancer survivors-a systematic review of quantitative studies. Psycho Oncol. 22, 1-11.

Lebel, S., Ozakinci, G., Humphris, G., Mutsaers, B., Thewes, B., Prins, J., Dinkel, A., Butow, P., University of Ottawa Fear of Cancer Recurrence Colloquium, a, 2016. From normal response to clinical problem: definition and clinical features of fear of cancer recurrence. Support. Care Canc. 24, 3265-3268.

Lee, S., Cook, E.F., Soiffer, R., Antin, J.H., 2002. Development and validation of a scale to measure symptoms of chronic graft-versus-host disease. Biol. Blood Marrow Transplant. 8, 444-452.

Lovibond, S.L.P., 1996. Manual for the Depression Anxiety Stress Scales. Psychology Foundation of Australia, Kensington.

Majhail, N.S., Farnia, S.H., Carpenter, P.A., Champlin, R.E., Crawford, S., Marks, D.I., Omel, J.L., Orchard, P.J., Palmer, J., Saber, W., Savani, B.N., Veys, P.A., Bredeson, C. N., Giralt, S.A., LeMaistre, C.F., 2015. Indications for autologous and allogeneic hematopoietic cell transplantation: guidelines from the American society for blood and marrow transplantation. Biol. Blood Marrow Transplant. 21, 1863-1869.

McDowell, M.E., Occhipinti, S., Ferguson, M., Dunn, J., Chambers, S.K., 2010. Predictors of change in unmet supportive care needs in cancer. Psycho Oncol. 19, 508-516.

McQuellon, R.P., Russell, G.B., Cella, D.F., Craven, B.L., Brady, M., Bonomi, A., Hurd, D. D., 1997. Quality of life measurement in bone marrow transplantation: development of the functional assessment of cancer therapy-bone marrow transplant (FACTHSCT) scale. Bone Marrow Transplant. 19, 357-368.

Mehnert, A., Koch, U., Sundermann, C., Dinkel, A., 2013. Predictors of fear of recurrence in patients one year after cancer rehabilitation: a prospective study. Acta Oncol. 52, 1102-1109.

Mehta, S.S., Lubeck, D., Pasta, D.J., Litwin, M.S., 2003. Fear of cancer recurrence in patients undergoing definitive treatment for prostate cancer: results from CaPSURE. J. Urol. 170, 1931-1933. 
Minstrell, M., Winzenberg, T., Rankin, N., Hughes, C., Walker, J., 2008. Supportive care of rural women with breast cancer in Tasmania, Australia: changing needs over time. Psycho Oncol. 17, 58-65.

Mohty, B., Mohty, M., 2011. Long-term complications and side effects after allogeneic hematopoietic stem cell transplantation: an update. Blood Canc. J. 1, e16.

Morris, B., Wilson, B., Chambers, S., 2013. Newfound compassion after prostate cancer: a psychometric evaluation of additional items in the posttraumatic growth inventory. Support. Care Canc. 21, 3371-3378.

Pavletic, S.Z., Martin, P., Lee, S.J., Mitchell, S., Jacobsohn, D., Cowen, E.W., Turner, M L., Akpek, G., Gilman, A., McDonald, G., Schubert, M., Berger, A., Bross, P., Chien, J. W., Couriel, D., Dunn, J.P., Fall-Dickson, J., Farrell, A., Flowers, M.E., Greinix, H., Hirschfeld, S., Gerber, L., Kim, S., Knobler, R., Lachenbruch, P.A., Miller, F.W. Mittleman, B., Papadopoulos, E., Parsons, S.K., Przepiorka, D., Robinson, M. Ward, M., Reeve, B., Rider, L.G., Shulman, H., Schultz, K.R., Weisdorf, D., Vogelsang, G.B., 2006. Measuring therapeutic response in chronic graft-versus-host disease: national institutes of health consensus development project on criteria for clinical trials in chronic graft-versus-host disease: IV. Response criteria working group report. Biol. Blood Marrow Transplant 12, 252-266.

Peteet, J.R., 2000. Cancer and the meaning of work. Gen. Hosp. Psychiatr. 22, 200-205.

Polinsky, M.L., 1994. Functional status of long-term breast cancer survivors: demonstrating chronicity. Health Soc. Work 19, 165-173.

Rabin, C., Leventhal, H., Goodin, S., 2004. Conceptualization of disease timeline predicts posttreatment distress in breast cancer patients. Health Psychol. 23, 407-412.

Rasmussen, D.M., Elverdam, B., 2008. The meaning of work and working life after cancer: an interview study. Psycho Oncol. 17, 1232-1238.

Sarkar, S., Scherwath, A., Schirmer, L., Schulz-Kindermann, F., Neumann, K., Kruse, M., Dinkel, A., Kunze, S., Balck, F., Kroger, N., Koch, U., Mehnert, A., 2014a. Fear of recurrence and its impact on quality of life in patients with hematological cancers in the course of allogeneic hematopoietic SCT. Bone Marrow Transplant. 49, $1217-1222$.

Sarkar, S., Scherwath, A., Schirmer, L., Schulz-Kindermann, F., Neumann, K., Kruse, M., Dinkel, A., Kunze, S., Balck, F., Kroger, N., Koch, U., Mehnert, A., 2014b. Fear of recurrence and its impact on quality of life in patients with hematological cancers in the course of allogeneic hematopoietic SCT. Bone Marrow Transplant. 49, 1217-1222.

Serna, D.S., Lee, S.J., Zhang, M.J., Baker, K.S., Eapen, M., Horowitz, M.M., Klein, J.P., Rizzo, J.D., Loberiza Jr., F.R., 2003. Trends in survival rates after allogeneic hematopoietic stem-cell transplantation for acute and chronic leukemia by ethnicity in the United States and Canada. J. Clin. Oncol. 21, 3754-3760.

Simard, S., Savard, J., 2009. Fear of Cancer Recurrence Inventory: development and initial validation of a multidimensional measure of fear of cancer recurrence. Support. Care Canc. 17, 241-251.

Simard, S., Savard, J., Ivers, H., 2010a. Fear of cancer recurrence: specific profiles and nature of intrusive thoughts. J Cancer Surviv 4, 361-371.

Simard, S., Savard, J., Ivers, H., 2010. Fear of cancer recurrence: specific profiles and nature of intrusive thoughts. J Cancer Survivor 4, 361-371.

Skaali, T., Fossa, S.D., Bremnes, R., Dahl, O., Haaland, C.F., Hauge, E.R., Klepp, O., Oldenburg, J., Wist, E., Dahl, A.A., 2009. Fear of recurrence in long-term testicular cancer survivors. Psycho Oncol. 18, 580-588.

Stanton, A.L., Danoff-Burg, S., Huggins, M.E., 2002. The first year after breast cancer diagnosis: hope and coping strategies as predictors of adjustment. Psycho Oncol. 11, 93-102.

Tedeschi, R., Calhoun, L., 1996. The posttraumatic growth inventory: measuring the positive legacy of trauma. J. Trauma Stress 9, 455-471.

Thewes, B., Butow, P., Zachariae, R., Christensen, S., Simard, S., Gotay, C., 2012a. Fear of cancer recurrence: a systematic literature review of self-report measures. Psycho Oncol. 21, 571-587.

Thewes, B., Butow, P., Zachariae, R., Christensen, S., Simard, S., Gotay, C., 2012b. Fear of cancer recurrence: a systematic literature review of self-report measures. Psycho Oncol. 21, 571-587.

van de Wal, M., van Oort, I., Schouten, J., Thewes, B., Gielissen, M., Prins, J., 2016. Fear of cancer recurrence in prostate cancer survivors. Acta Oncol. 55, 821-827.

Vickberg, S.M., 2003. The concerns about recurrence scale (CARS): a systematic measure of women's fears about the possibility of breast cancer recurrence. Ann. Behav. Med. $25,16-24$. 